\title{
Diagnosing Latent Tuberculosis in Immunocompromised Patients Measuring Blood IP-10 Production Capacity: An Analysis of Chronic Renal Failure Patients
}

\author{
Gulsah Gunluoglu ${ }^{1}$, Ekrem Cengiz Seyhan ${ }^{2}$, Rumeyza Kazancioglu ${ }^{3}$, Zeki Gunluoglu ${ }^{4}$, \\ Nurdan Simsek Veske ${ }^{1}$, Esra Ertan Yazar ${ }^{1}$ and Sedat Altin ${ }^{1}$
}

\begin{abstract}
Objective Patients undergoing haemodialysis for chronic renal failure-hemodialysis (CRF-HD) are at risk of latent tuberculosis infection (LTBI). The effectiveness of using blood IP-10 production capacity to diagnose LTBI in CRF-HD patients was analysed.

Methods The study enrolled $50 \mathrm{CRF}-\mathrm{HD}$ patients. Interferon- $\gamma$ release assay (IGRA) was done using QuantiFERON-TB Gold In Tube (QFG-IT) system. Blood IP-10 production capacity was measured using the QFG-IT system tubes. Tuberculin skin testing (TST) was performed on the same day and the test results were compared.

Results TST turned out to be positive in $36.4 \%$ of the patients and QFG-IT in $54 \%$ of them. After stimulation with specific tuberculosis antigens, blood IP-10 levels increased noticeably. The antigen-stimulated blood IP-10 level was significantly higher in patients who were either TST or QFG-IT positive than in patients whose tests were negative $(\mathrm{p}=0.0001)$. Using $4.02 \mathrm{pg} / \mathrm{mL}$ as the threshold for stimulated blood logtransformed IP-10 level, good agreement was observed between IP-10 and QFG-IT results ( $\kappa=1)$.

Conclusion Blood IP-10 level, which can be measured simply, provides results equivalent to IGRAs for the diagnosis of LTBI in CRF-HD patients.
\end{abstract}

Key words: latent tuberculosis infection, chronic renal failure, IP-10, IGRA, TST

(Intern Med 54: 465-472, 2015)

(DOI: 10.2169/internalmedicine.54.3245)

\section{Introduction}

According to the World Health Organisation, one-third of the global population is infected with Mycobacterium tuberculosis $(M t b)(1)$. Fortunately, in most people, the infection is kept under control by immune defence and does not generate active disease (latent tuberculosis infection, LTBI).

The classical method used to diagnose LTBI is tuberculin skin testing (TST). This test has various disadvantages. Menzies conducted a meta-analysis and found that the reported sensitivity of TST in diagnosing LTBI is $71 \%$, and that the specificity of TST in diagnosing LTBI is very low
(56\%) among individuals who have had the Bacillus Calmette-Guérin (BCG) vaccination (2).

One of the major developments in the diagnosis of TB in the last decade is the introduction of new-generation tests known as interferon- $\gamma$ release assays (IGRAs). These measure the in-vitro $\mathrm{T}$ cell response to $M t b$-specific antigens (3). However, its results are more specific than those of TST, because the testing uses two $M t b$-specific antigens: Early Secreted Antigenic Target (ESAT)-6 and Culture Filtrate Protein (CFP)-10 plus TB7.7. These antigens are not present in BCG and most non-tuberculous mycobacteria, and are used instead of purified protein derivative (PPD) using in TST $(4,5)$. In Menzies' meta-analysis, the specificity of the

\footnotetext{
${ }^{1}$ Department of Chest Diseases, Yedikule Teaching Hospital for Chest Diseases and Thoracic Surgery, Turkey, ${ }^{2}$ Department of Chest Diseases, Medipol University Faculty of Medicine, Turkey, ${ }^{3}$ Department of Nephrology, Bezmialem Vakif University Faculty of Medicine, Turkey and ${ }^{4}$ Department of Thoracic Surgery, Faculty of Medicine, Medipol University, Turkey

Received for publication May 8, 2014; Accepted for publication July 21, 2014

Correspondence to Dr. Gulsah Gunluoglu, gunluoglugulsah@yahoo.com.tr
} 
QuantiFERON test (QFT), which is one of two commercial IGRAs, for detecting LTBI was $96 \%$ among those with the BCG vaccination and $100 \%$ among those without vaccination (2). However, IGRAs do not achieve a significant improvement in sensitivity for TB diagnosis compared to TST.

Antigen-presenting cells (APCs) secrete a $10-\mathrm{kDa}$ interferon (IFN)- $\gamma$ inducible protein (IP-10) upon stimulation with IFN- $\gamma$ and various cytokines (6). IFN- $\gamma$ is the 10th member of the CXC chemokine family. IFN- $\gamma$, which is secreted by Th1 lymphocytes that encounter the antigen on the surface of APCs, activates T cells and macrophages, while inducing the expression of IP-10 by APCs $(7,8)$. Then, IP10 interacts with CXC chemokine receptor-3 (CXCR-3) triggering the chemotaxis of activated $\mathrm{T}$ lymphocytes and macrophages $(9,10)$. This small $(2.5$ times smaller than IFN- $\gamma$ ) molecule (11), which is essentially a chemokine, is the major inducer of pro-inflammatory immune responses (12). Therefore, it can be used as an indicator of the cell-mediated immune response (8). In 2006, Ruhwald found that the IP-10 level was very high in QFT test tube supernatants from TB patients and low in those from healthy individuals (13). Consequently, the IP-10 production capacity is thought to be another way of assessing cellular immunity in TB patients (14). Studies have demonstrated that IP-10 can be induced by specific antigens in TB patients (11). A few limited case-control studies revealed that IP-10 is a potential biomarker in the diagnosis of TB $(15,16-19)$. The blood IP10 production capacity is reportedly as effective as QFT in the diagnosis of $\operatorname{LTBI}(17,20,21)$. Moreover, because it is secreted in greater amounts, it might be easier to measure than IFN- $\gamma$.

The risk of LTBI activation is high in immunocompromised persons, so it is recommended that they be screened for LTBI (22). The sensitivity of TST in the diagnosis of TB is lower in immunocompromised individuals than in the normal population (23). IGRAs are known to be superior to TST for detecting TB or LTBI in this population (24). However, the sensitivity of IGRAs is also inadequate $(11,25)$, perhaps because the level of IFN- $\gamma$ expressed is even lower in immunocompromised persons and cannot be detected (26). Therefore, a marker that is expressed at a higher level might be more sensitive for diagnosis, particularly among this population $(2,20)$.

At least $50 \%$ of chronic renal failure patients under haemodialysis therapy chronic renal failure-hemodialysis (CRF-HD) patients who have active TB may have anergy to TST due to their weakened immune system $(27,28)$. Therefore, TST is not sufficiently reliable to rule out the possibility of active TB or LTBI. Among CRF-HD patients at risk for LTBI, many more are IGRA positive than TST positive (29). Nevertheless, the sensitivity of IGRAs is still approximately $70 \%$ at most (30). Hence, IGRAs are far from being perfect for LTBI screening of CRF-HD patients.

As IP-10 is produced in greater amounts than IFN- $\gamma$, it might be more useful in the diagnosis of TB or LTBI in patients with limited immune functions (11). This study ana- lysed the blood IP-10 production capacity in CRF-HD patients as an immunocompromised patient group to test the effectiveness of using IP-10 level to diagnose LTBI in immunocompromised patients.

\section{Materials and Methods}

\section{Recruitment}

The study was performed in accordance with the principles of the Declaration of Helsinki and approved by the ethics committee of Yedikule Chest Diseases and Thoracic Surgery Training and Research Hospital. Written informed consent was obtained from each enrolled participant.

The study group included CRF patients who underwent regular haemodialysis between September 2011 and November 2011. The 50 patients who agreed were included in the study. Blood was drawn from these patients to determine their IFN- $\gamma$ and IP-10 levels. All of the patients also underwent TST on the same day.

\section{Study population}

The patients included 22 females and 28 males with a mean age of 62.2 years. They had undergone thrice weekly haemodialysis for an average of 54.9 months. Fifteen patients were diabetics. None of the patients had human immunodeficiency virus (HIV) infection. None of the patients exhibited any active TB symptomatology or had radiological findings of active disease.

\section{Assessing previous exposure and risk factors}

Information about other illnesses, history of TB infection, and known exposure to TB patients was collected via interviews. Chest X-rays were reviewed routinely by the private dialysis centre. BCG vaccination status was ascertained from a self-reported vaccination history or the detection of a BCG vaccination scar on visual inspection. Twenty-one patients had a BCG scar or vaccination history.

\section{LTBI risk factors}

Fourteen of the patients had one or more LTBI risk factors. Seven patients had previously recovered from TB. Four patients had a history of exposure to a TB patient. In three patients who had not had TB and had no history of exposure to a TB patient, the chest X-rays showed radiological changes that could be associated with previous TB (dense pulmonary nodules with or without visible calcification in the hilar area or upper lobes or pleural scarring).

\section{Tuberculin skin testing}

Tuberculin skin testing was performed using the Mantoux method intradermally on the volar surface of the forearm contralateral to the arteriovenous shunt with $0.1 \mathrm{~mL}$ (5 TU) of PPD (Intervax Biologicals, Markham, Canada). Responses were read on the patient's next regularly scheduled HD day, 48-72 hours after TST. As suggested by the Cen- 
Table 1. Relationship between BCG Vaccination and TST or QFG-IT Results

\begin{tabular}{lrr}
\hline & $\begin{array}{l}\text { BCG vaccinated patients, } \mathrm{n} \\
(\%)\end{array}$ & $\begin{array}{l}\text { BCG not vaccinated patients, } \mathrm{n} \\
(\%)\end{array}$ \\
\hline TST result & & \\
Negative & $17(68)$ & $11(57.9)$ \\
Positive & $8(32)$ & $8(42.1)$ \\
QFG-IT result & & $10(47.6)$ \\
Negative & $13(44.8)$ & $11(52.4)$ \\
$\quad$ Positive & $16(55.2)$ & \\
\hline
\end{tabular}

tres for Disease Control and Prevention (CDC) for CRF patients (31), induration $\geq 10 \mathrm{~mm}$ was considered positive for the CRF patients.

\section{Whole blood stimulation}

For the test, $1 \mathrm{~mL}$ of whole blood was drawn in each of three Vacutainer tubes provided with the QuantiFERON-TB Gold In-tube (QFG-IT) system (Cellestis, Carnegie, Australia), a new-generation QFT test. These tubes are already pre-coated with saline (negative control), the ESAT-6 and CFP10 peptides and TB 7.7 antigen (antigen stimulated), and phytohaemagglutinin (positive mitogen control). The tubes were mixed and incubated at $37^{\circ} \mathrm{C}$ for $20-24$ hours, when the plasma was harvested and frozen until further analysis. This plasma provided the unstimulated, antigenstimulated, and mitogen-stimulated supernatant samples.

\section{IFN- $\gamma$ quantification}

IFN- $\gamma$ was measured by an enzyme-linked immunosorbent assay (ELISA) using the QFG-IT test, according to the manufacturer's instructions (32). A result of $\geq 0.35 \mathrm{IU} / \mathrm{mL}$ IFN- $\gamma$ in the TB antigen tube minus the negative control tube was considered a positive result. If the level was less than this and the mitogen control was positive $(\geq 0.5 \mathrm{IU} /$ $\mathrm{mL}$ ), a negative result was recorded. If the level in both the TB antigen and mitogen tubes was less than the threshold for positive, or the level in the nil tube was $>8.0 \mathrm{IU} / \mathrm{mL}$, then an indeterminate result was recorded.

\section{IP-10 quantification}

The RayBio Human IP-10 ELISA kit (RayBiotech, Norcross, USA) was used to quantify IP-10 (33). This kit has a microplate with 96 wells pre-coated with a specific monoclonal antibody for human IP-10. IP-10 supernatant levels were measured using a sandwich ELISA performed according to the manufacturer's instructions (http://www.raybiotech. com/files/manual/ELISA/ELHIP10-001.pdf). Samples were diluted 1:2. First, 50- $\mu \mathrm{L}$ samples were taken from each of the three QFG-IT test tubes and placed in the wells, which were covered and incubated for 2.5 hours at room temperature. After washing four times, $50 \mu \mathrm{L}$ of prepared biotin antibody were added to each well and incubated for 1 hour at room temperature. The washing procedure was repeated. Then, $100 \mu \mathrm{L}$ of streptavidin were added to the wells and incubated for 45 minutes at room temperature. After the washing procedure, $50 \mu \mathrm{L}$ of TMB One-Step Substrate Reagent were added to each well and incubated for 30 minutes at room temperature in the dark. Finally, $100 \mu \mathrm{L}$ of Stop Solution were added to each well and read immediately at $450 \mathrm{~nm}$ in an ELISA reader.

IP-10 measurement values are very high (up to $60,000 \mathrm{pg} /$ $\mathrm{mL}$ ). To improve interpretability, the results were transformed logarithmically (common logarithm).

\section{Statistical analyses}

All statistical analyses were performed using Statistical Package for the Social Sciences (SPSS) for Windows (ver. 11.0, SPSS, Chicago, USA). Variables are presented as the medians. Kappa statistic was used to define agreement between two measurements for categorical variables (scaled between 0 and 1). Mann-Whitney $\mathrm{U}$ test was used to compare median values and the chi-square or Fisher's exact test was used to compare frequencies. Wilks' lambda test was used to find the significance of the differences between repeated measures. All tests were two-tailed, and $p<0.05$ was considered significant.

\section{Results}

\section{TST Results}

Tuberculin skin testing was performed in 44 patients who agreed to it. The induration diameter averaged 13.5 (range $10-20) \mathrm{mm}$. The test was positive in 16 of the patients (36.4\%) and negative in $28(63.6 \%)$. No differences were observed in the prevalence of diabetes between the patients with positive and negative test results $(\mathrm{p}=0.31)$. TST was positive in $32 \%$ of those who had the BCG vaccine, but no differences appeared in the prevalence of BCG vaccination between the patients with positive and negative test results (Table 1) $(\mathrm{p}=0.54)$. TST was positive in $54.5 \%$ of those who had risk factors for LTBI, but the relationship between the test result and LTBI risk $(\kappa=0.21)$ was not significant $(\mathrm{p}=$ 0.15) (Table 2).

\section{QFG-IT results}

The QFG-IT test was administered to all of the patients: it was positive in $27(54 \%)$, negative in $23(46 \%)$, and indeterminate in none $(0 \%)$. No differences were observed in the prevalence of diabetes between the patients with positive and negative test results $(\mathrm{p}=1)$. The test was positive in $55.2 \%$ of those vaccinated with BCG, but no differences appeared in the prevalence of BCG vaccination between the patients with positive and negative test results $(\mathrm{p}=1)$ (Table 1). The test was positive in $71.4 \%$ of those who had risk factors for LTBI, but the relationship between the test result and LTBI risk factors was at the $\kappa=0.19$ level and was not significant $(\mathrm{p}=0.12)$ (Table 2).

Table 3 compares the results of the 44 patients who underwent both TST and the QFG-IT. While $18.8 \%$ of the patients whose TST was positive had a negative QFG-IT re- 
Table 2. Relationship between LTBI Risk Factors and TST or QFGIT Results

\begin{tabular}{|c|c|c|c|c|c|}
\hline & \multicolumn{5}{|c|}{ Risk factor } \\
\hline & $\begin{array}{l}\text { Previous TB } \\
\text { infection, } \mathrm{n}\end{array}$ & $\begin{array}{l}\text { TB contact, } \\
\mathrm{n}\end{array}$ & $\begin{array}{l}\text { TB sequela } \\
\text { in chest X- } \\
\text { ray, } n\end{array}$ & $\begin{array}{l}\text { Present,total, } \\
\mathrm{n}(\%)\end{array}$ & $\begin{array}{l}\text { Absent,n } \\
(\%)\end{array}$ \\
\hline \multicolumn{6}{|l|}{ TST result } \\
\hline Negative & 1 & 3 & 1 & $5(45.5)$ & $23(69.7)$ \\
\hline Positive & 4 & 1 & 1 & $6(54.5)$ & $10(30.3)$ \\
\hline \multicolumn{6}{|l|}{ QFG-IT result } \\
\hline Negative & 1 & 2 & 1 & $4(28.6)$ & $19(52.8)$ \\
\hline Positive & 6 & 2 & 2 & $10(71.4)$ & $17(47.2)$ \\
\hline
\end{tabular}

Table 3. Comparison of TST and QFG-IT Test Results

\begin{tabular}{lrr}
\hline & QFG-IT positive & QFG-IT negative \\
\hline TST positive & $13(81.2 \%)$ & $3(18.8 \%)$ \\
TST negative & $9(32.1 \%)$ & $19(67.9 \%)$ \\
\hline
\end{tabular}

sult, $32.1 \%$ of the patients whose TST was negative had a positive QFG-IT result. For TST/QFG-IT agreement $\kappa=0.46$ $(\mathrm{p}=0.002)$.

\section{IP-10 measurements}

Due to the non-Gaussian distribution, median values are presented. Table 4 lists the median log-transformed IP-10 levels of unstimulated (baseline), antigen-stimulated, and mitogen-stimulated blood. The unstimulated and mitogenstimulated blood log-transformed IP-10 levels of the patients that were positive for either TST or QFG-IT did not differ from those of the patients with negative results (MannWhitney $U$-test, Table 4). All of the patients responded to mitogen. The post-mitogen IP-10 levels of five patients were lower than their antigen-stimulated blood IP-10 levels.

When stimulated with specific TB antigens, blood IP-10 levels rose markedly compared to before stimulation (Wilks' lambda, $\mathrm{p}=0.0001)$. The increase was significant in all patients who were TST negative $(\mathrm{p}=0.002)$, TST positive $(\mathrm{p}=$ $0.0001)$, or QFG-IT negative $(\mathrm{p}=0.021)$ or positive $(\mathrm{p}=$ 0.0001). While the median log transformed IP-10 level in stimulated blood of patients with no risk factors for LTBI was $3.96 \mathrm{pg} / \mathrm{mL}$, it was $4.64 \mathrm{pg} / \mathrm{mL}$ for patients with risk factors $(\mathrm{p}=0.039$, Mann-Whitney $U$-test $)$. The antigenstimulated blood IP-10 level was significantly higher in patients who were either TST or QFG-IT positive than in patients whose tests were negative $(\mathrm{p}=0.0001$, Mann-Whitney $U$-test, Table 4).

Because there is no gold standard for diagnosing LTBI, a cut-off value for the IP-10 level in diagnosing LTBI could not been analysed. However, the maximum antigenstimulated blood log transformed IP-10 level was $4.02 \mathrm{pg} /$ $\mathrm{mL}$ in patients with a negative QFG-IT test, whereas the minimum level was $4.14 \mathrm{pg} / \mathrm{mL}$ in patients with a positive QFG-IT test (Fig. 1). Taking a log transformed IP-10 level in stimulated blood of $4.02 \mathrm{pg} / \mathrm{mL}$ as the threshold for the
IP-10 test result, good agreement $(\kappa=1)$ was reached between the QFG-IT and IP-10 tests; all of the patients whose stimulated blood log transformed IP-10 level exceeded 4.02 $\mathrm{pg} / \mathrm{mL}$ had a positive QFG-IT test. In comparison, TST was negative in nine patients with stimulated blood log transformed IP-10 levels higher than $4.02 \mathrm{pg} / \mathrm{mL}$, three of whom had either risk factor for LTBI or BCG vaccination. While the QFG-IT results of all of the patients with a stimulated blood log transformed IP-10 level less than $4.02 \mathrm{pg} / \mathrm{mL}$ were negative, TST gave positive results in three of these patients. Two of these had no risk factors for LTBI, but had been vaccinated with BCG; the other patient had not been vaccinated with BCG, but had active TB (Fig. 2). When $4.02 \mathrm{pg} / \mathrm{mL}$ in stimulated blood was used as the threshold value for the IP-10 test, a moderate relationship $(\kappa=0.46, \mathrm{p}=$ 0.002 ) appeared between the TST and IP-10 results. The IP10 levels of the patients who had positive and negative TST results intersected markedly on the intersection graph (Fig. 3).

\section{Discussion}

Blood IP-10 levels are high in individuals with household TB contact $(17,34)$. Additionally, blood IP-10 levels in individuals with LTBI increase with stimulation $(17,35)$. Consequently, blood IP-10 level might be valuable for diagnosing LTBI. In this study, good agreement was found between the QFG-IT and IP-10 results in CRF patients. Therefore, the QFG-IT test or stimulated blood IP-10 level could be used to diagnose LTBI in immunocompromised individuals with CRF. Although it is impossible to state that these tests are more accurate than TST because there is no gold standard for detecting LTBI, the larger number of positive CRF patients using the QFG-IT test or IP-10 level in stimulated blood makes these tests seem more precise than TST.

According to the government sources, the estimated prevalence of LTBI among healthy population in our country is $25 \%$ (36). The rate of LTBI can be as high as $70 \%$ in CRF patients (37). In our series, however, LTBI was detected in $36.4 \%$ of the CRF patients based on TST. This rate is only slightly higher than the LTBI rate expected in the healthy population. Therefore, the LTBI rate found in CRF patients with TST is likely lower than the actual rate. One 
Table 4. Results of Blood Log-transformed IP-10 Level Measurements

\begin{tabular}{|c|c|c|c|c|c|c|c|c|c|}
\hline $\begin{array}{l}\text { Median log- } \\
\text { transformed IP- } \\
10 \text { level in }\end{array}$ & $\begin{array}{l}\text { TST } \\
\text { negative } \\
\text { patients }\end{array}$ & $\begin{array}{l}\text { TST } \\
\text { positive } \\
\text { patients }\end{array}$ & $\mathrm{p}^{*}$ & $\begin{array}{l}\text { QFG-IT } \\
\text { negative } \\
\text { patients }\end{array}$ & $\begin{array}{l}\text { QFG-IT } \\
\text { positive } \\
\text { patients }\end{array}$ & $\mathrm{p}^{*}$ & $\begin{array}{l}\text { TST or } \\
\text { QFG-IT } \\
\text { negative } \\
\text { patients }\end{array}$ & $\begin{array}{l}\text { TST or } \\
\text { QFG-IT } \\
\text { positive } \\
\text { patients }\end{array}$ & $\mathrm{p}^{*}$ \\
\hline $\begin{array}{l}\text { Unstimulated } \\
\text { blood, pg/mL }\end{array}$ & 1.79 & 2.33 & 0.18 & 1.81 & 2.22 & 0.53 & 1.77 & 2.20 & 0.33 \\
\hline $\begin{array}{l}\text { Mitogen } \\
\text { stimulated } \\
\text { blood, pg/mL }\end{array}$ & 4.77 & 4.78 & 0.33 & 4.78 & 4.78 & 0.76 & 4.78 & 4.78 & 0.77 \\
\hline $\begin{array}{l}\text { Antigen } \\
\text { stimulated } \\
\text { blood, pg/mL }\end{array}$ & 3.03 & 4.54 & 0.004 & 1.91 & 4.63 & 0.0001 & 1.90 & 4.59 & 0.0001 \\
\hline
\end{tabular}

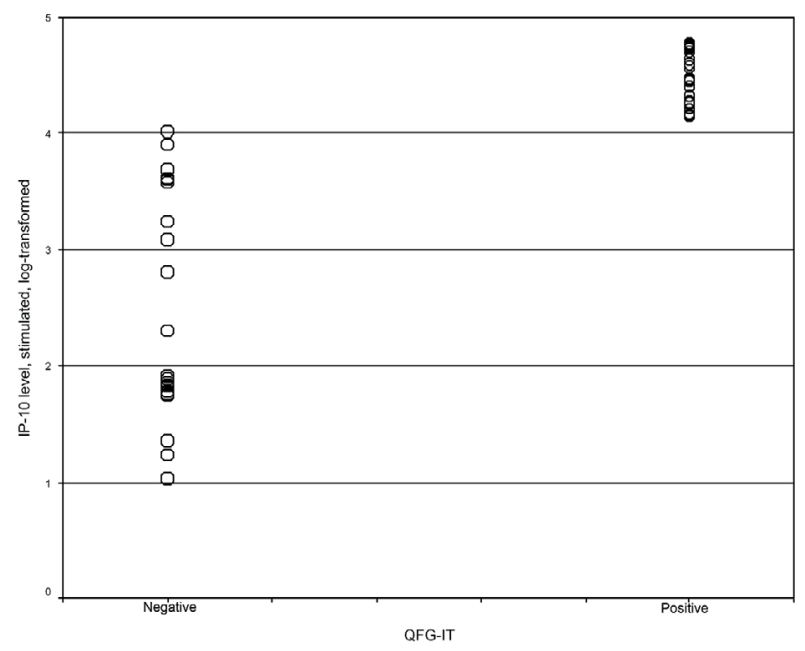

Figure 1. Intersection graph shows patients' QFG-IT results according to IP-10 level in stimulated blood.

possible reason for this is that the anergy rate is higher in CRF patients. The reported rate of anergy to TST can exceed $50 \%$ in CRF patients with active TB $(27,28)$; this may be caused by the immunosuppression that occurs in CRF patients. We found more positive patients $(54 \%)$ using the QFG-IT test or stimulated blood IP-10 level than with TST. If the results of these tests are correct, this rate suggests a high LTBI risk in CRF patients.

One of the disadvantages of TST in the diagnosis of TB or LTBI is the false positive results caused by BCG vaccination $(4,5,38,39)$. In our series, we found less positivity with TST than with QFG-IT, even among individuals vaccinated with BCG. This finding may be related to the anergy or immunosuppression expected in CRF patients. Anergy disguises not only true positivity, but also false positivity, which might explain why more positivity in immunocompromised people has been reported with IGRAs than with TST (40).

Some studies have shown that IGRAs are better correlated with LTBI risk factors than TST (30). The same result has been obtained in CRF patients $(41,42)$. Passalent et al. reported that IGRA was positive in more than $70 \%$ of CRF patients with risk factors for LTBI, while TST gave positive results in roughly $20 \%$ (29). We found more positivity with QFG-IT testing (or the IP-10 level in stimulated blood) than with TST in CRF patients who had LTBI risk factors (71.4\% vs. $54.5 \%$ ). This finding suggests that the QFG-IT (or IP-10) test is affected less by immunosuppression. However, because the rate of positive results with the QFG-IT (or IP-10) test was also high among those who did not have any risk factors for LTBI $(47.2 \%$ vs. $30.3 \%)$, good agreement was not obtained between the presence of LTBI risk factors and the test results. The IGRA-TST agreement in the diagnosis of LTBI is reportedly generally moderate $(42,43)$, and our findings support this.

In our series, the increase in blood IP-10 levels after stimulation with specific TB antigens was high. A limited rise in IP-10 levels after stimulation can be observed in the blood of healthy people, probably due to non-specific effects of the antigens $(13,16)$. However, blood IP-10 levels rise distinctly after stimulation with specific antigens in active TB patients compared with healthy people $(13,35)$. Blood IP-10 levels also rise with stimulation in individuals with LTBI $(17,35)$. We observed a good blood IP-10 response upon stimulation with specific TB antigens in CRF-HD patients. Therefore, blood IP-10 level is not affected by immunosuppression. Consequently, blood IP-10 measurement might useful in the diagnosis of LTBI in immunocompromised people.

Because the IP-10 and QFG-IT results were the same when the threshold value of $4.02 \mathrm{pg} / \mathrm{mL}$ for the log transformed IP-10 level in stimulated blood was used, the relationship between IP-10 level and LTBI risk factors is the same as the relationship between QFG-IT and the risk factors. The presence of patients who have LTBI risk factors and a negative TST result, but a positive IP-10 or QFG-IT test result, suggests the superiority of the QFG-IT or IP-10 test over TST. Because two-thirds of the patients who were IP-10 or QFG-IT negative and TST positive had been vaccinated with BCG, this suggests that the TST result is inaccurate and the QFG-IT or IP-10 result is more accurate.

The reported rate of positive results in healthy people is $2 \%$ for IP-10 and $0 \%$ for IGRA tests $(6,21)$. In contrast, with regard to LTBI, IP-10 was reportedly positive in $35 \%$ of the patients suspected of LTBI due to risk factors and 


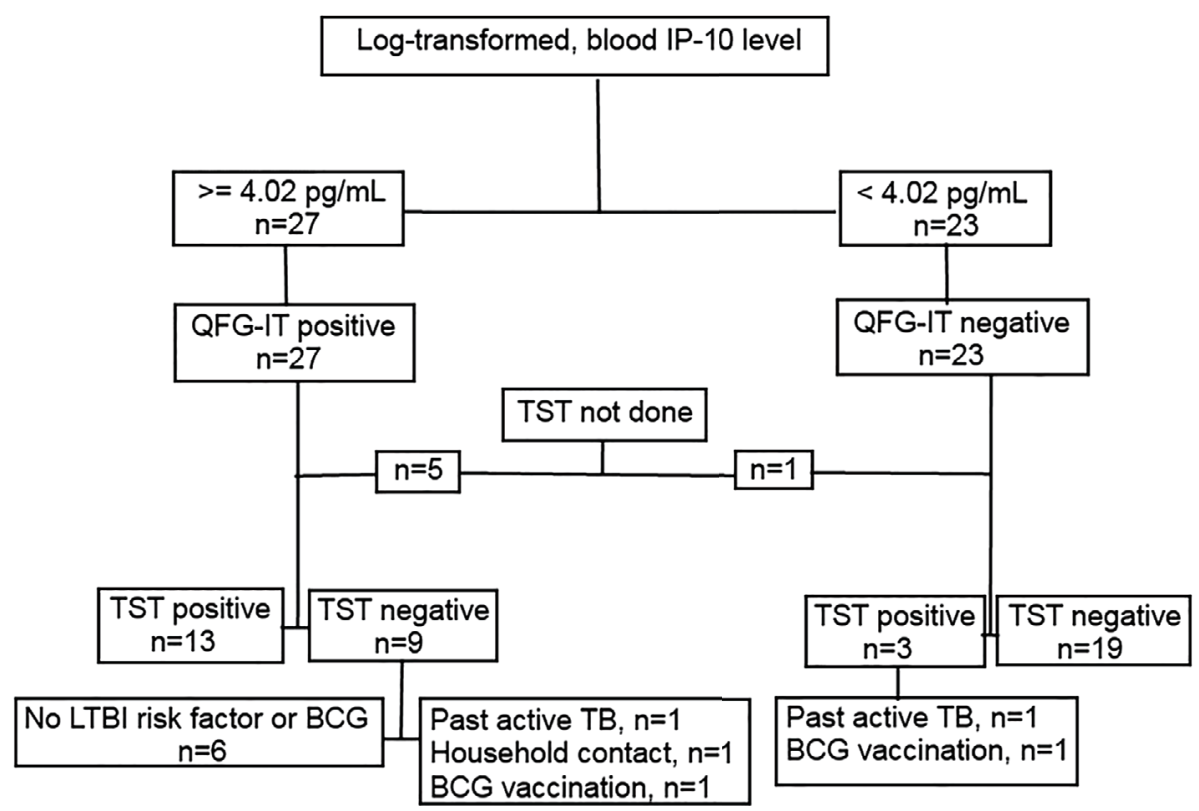

Figure 2. Relationship between IP-10 levels in stimulated blood and QFG-IT and TST results

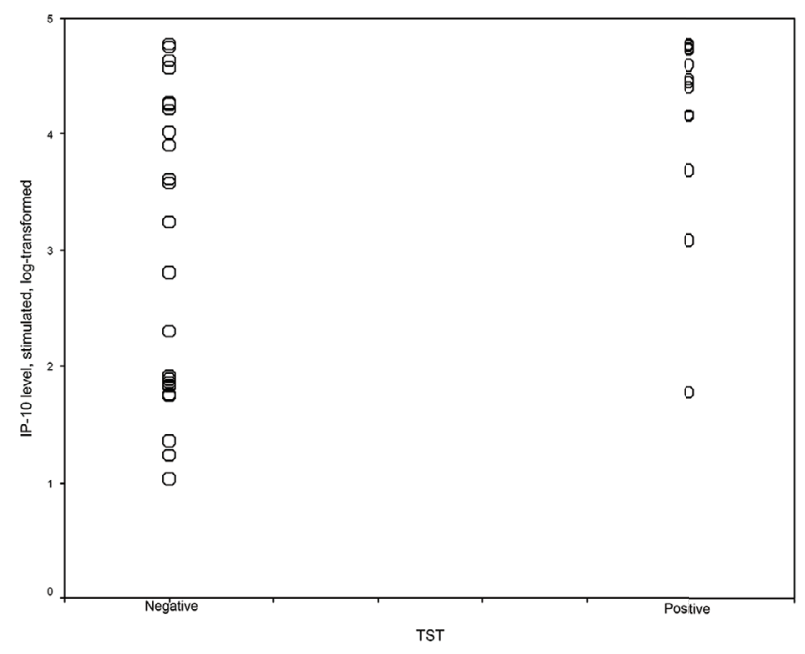

Figure 3. Intersection graph shows patients' TST results according to IP-10 levels in stimulated blood.

QFT was positive in $27 \%$ of them in a European multicentre study $(\mathrm{p}<0.05)(6)$. In the diagnosis of LTBI, more individuals have positive results with IP-10 measurements than IGRAs. This higher rate might be because the blood IP-10 level is higher than the IFN- $\gamma$ level (6), making it more easily detected, and meaning that its sensitivity is greater than that of IGRA.

Conversely, in immunocompromised individuals, the rate of increase in the blood IFN- $\gamma$ level is not as great, even after stimulation (19), whereas IP-10 is generated in greater amounts than IFN- $\gamma$. The blood IP-10 level was 29.1 times higher than the IFN- $\gamma$ level in those with TB (6). Therefore, it can be more useful for diagnosing TB among patients with limited immune functions (11). In fact, immunocompromised individuals have high blood IP-10 levels (44). In HIV patients, the blood IFN- $\gamma$ response declines noticeably, while the IP-10 response is affected less (20), resulting in a high IP-10 response to QFT antigens (14). The reason for this might be that IP-10 is secreted by macrophages rather than CD4 (12). No relationship has been found between the blood IP-10 level and amount of CD4 (19). In other words, the IP-10 level is not affected by decreases in the amount of CD4 (11).

However, we did not find that the IP-10 results were more useful among CRF patients, when compared with QFG-IT. Due to the very good correlation between the QFG-IT and IP-10 results, both methods seem to be equally accurate when diagnosing LTBI in CRF patients. Using IP10 in place of IGRA for these patients might have various operational advantages for the following reasons:

1. Because IP-10 is highly expressed,

a. It is easier to detect;

b. A small sample is sufficient as IP-10 mRNA can be obtained from a capillary blood sample taken from the fingertip (45); and

c. It can be measured using simple technology (lateral flow platform as in HIV and pregnancy tests) (6),

2. There is no need for a cold chain to transport it; it remains stable in dry blood or plasma (6), and can even be transported on filter paper.

Some problems with the IP-10 analysis remain unsolved. First, the analysis method (dilution) could not be standardised (15). Therefore, the variance (range 1,402-19,350) in the reported median IP-10 levels is large $(16-23,46)$. To date, no accepted threshold value has been determined for positive or indeterminate results. The log-transformed level of $4.02 \mathrm{pg} / \mathrm{mL}$ found in this study for a 1:2 dilution can serve a guideline for future research. 


\section{Conclusion}

Measurement of blood IP-10 levels after stimulation with specific TB antigens might be reliable for diagnosing LTBI in CRF-HD patients. Compared with IGRA, IP-10 measurement is equally successful among these patients.

\section{The authors state that they have no Conflict of Interest (COI).}

\section{Acknowledgement}

We want to thank Dr. Orhan Kaya Koksalan for his help in analyzing the blood samples.

\section{References}

1. WHO. Global tuberculosis control: surveillance, planning, financing. WHO Report 2005. WHO, Geneva, Switzerland, 2005: 1-247.

2. Menzies D, Pai M, Comstock G. Meta-analysis: new tests in the diagnosis of latent tuberculosis infection: areas of uncertainty and recommendations for research. Ann Intern Med 146: 340-354, 2007.

3. Andersen P, Munk ME, Pollock JM, Doherty TM. Specific immune-based diagnosis of tuberculosis. Lancet 356: 1099-1104, 2000.

4. Diel R, Goletti D, Ferrara G, et al. Interferon- $\gamma$ release assays for the diagnosis of latent Mycobacterium tuberculosis infection: a systematic review and meta-analysis. Eur Respir J 37: 88-99, 2011.

5. Pai M, Zwerling A, Menzies D. Systematic review: T-cell-based assays for the diagnosis of latent tuberculosis infection: an update. Ann Intern Med 149: 177-184, 2008.

6. Ruhwald M, Dominguez J, Latorre I, et al; TBNET. A multicentre evaluation of the accuracy and performance of IP-10 for the diagnosis of infection with M. tuberculosis. Tuberculosis 91: 260-267, 2011.

7. Groom JR, Luster AD. CXCR3 ligands: redundant, collaborative and antagonistic functions. Immunol Cell Biol 89: 207-215, 2011.

8. Chen J, Liu X. The role of interferon $g$ in regulation of CD4+ Tcells and its clinical implications. Cell Immunol 254: 85-90, 2009.

9. Farber JM. Mig and IP-10: CXC chemokines that target lymphocytes. J Leukoc Biol 61: 246-257, 1997.

10. Ferrero E, Biswas $P$, Vettoretto $\mathrm{K}$, et al. Macrophages exposed to Mycobacterium tuberculosisrelease chemokines able to re-cruit selected leukocytes subpopulations: focus on gammadelta cells. Immunology 108: 365-374, 2003.

11. Ruhwald M, Aabye MG, Ravn P. IP-10 release assays in the diagnosis of tuberculosis infection: current status and future directions. Expert Rev Mol Diagn 12: 175-187, 2012.

12. Moser B, Loetscher P. Lymphocyte traffic control by chemokines. Nat Immunol 2: 123-128, 2001.

13. Ruhwald M, Bjerregaard-Andersen $M$, Rabna $P$, Kofoed $K$, Eugen-Olsen J, Ravn P. IP-10/CXCL10 release is induced by incubation of whole blood from tuberculosis patients with ESAT-6, CFP10 and TB7.7. Microbes Infect 9: 806-812, 2007.

14. Kabeer BS, Sikhamani R, Raja A. Comparison of interferon gamma-inducible protein-10 and interferon gamma-based QuantiFERON TB Gold assays with tuberculin skin test in HIV-infected subjects. Diagn Microbiol Infect Dis 71: 236-243, 2011.

15. Lighter J, Rigaud M, Huie M, Peng CH, Pollack H. Chemokine IP-10: an adjunct marker for latent tuberculosis infection in children. Int J Tuberc Lung Dis 13: 731-736, 2009.

16. Whittaker E, Gordon A, Kampmann B. Is IP-10 a better biomarker for active and latent tuberculosis in children than IFN gamma? PLoS One 3: e3901, 2008.

17. Ruhwald M, Petersen J, Kofoed K, et al. Improving T-cell assays for the diagnosis of latent TB infection: potential of a diagnostic test based on IP-10. PLoS One 3: e2858, 2008.

18. Dheda K, van Zyl Smit R, Badri M, Pai M. T-cell interferongamma release assays for the rapid immunodiagnosis of tuberculosis: clinical utility in high-burden vs. low-burden settings. Curr Opin Pulm Med 15: 188-200, 2009.

19. Goletti D, Raja A, Syed Ahamed Kabeer B, et al. Is IP-10 an accurate marker for detecting $\mathrm{M}$. tuberculosis-specific response in HIV-infected persons? PLoS One 5: e12577, 2010.

20. Aabye MG, Ruhwald M, Praygod G. Potential of interferon- $\gamma$ inducible protein 10 in improving tuberculosis diagnosis in HIVinfected patients. Eur Respir J 36: 1488-1490, 2010.

21. Ruhwald M, Bodmer T, Maier C, et al. Evaluating the potential of IP-10 and MCP-2 as biomarkers for the diagnosis of TB. Eur Respir J 32: 1607-1615, 2008.

22. Canadian Tuberculosis Committee (CTC). Updated recommendations on interferon gamma release assays for latent tuberculosis infection. An Advisory Committee Statement (ACS). Can Commun Dis Rep 34 (ACS-6): 1-13, 2008.

23. American Thoracic Society. Targeted tuberculin testing and treatment of latent tuberculosis infection. MMWR Recomm Rep 49: 1$51,2000$.

24. Lalvani A, Pareek M. A 100 year update on diagnosis of tuberculosis infection. Br Med Bull 93: 69-84, 2010.

25. Raby E, Moyo M, Devendra A, et al. The effects of HIV on the sensitivity of a whole blood IFN-gamma release assay in Zambian adults with active tuberculosis. PLoS ONE 3: e2489, 2008.

26. Ruhwald M, Ravn P. Immune reconstitution syndrome in tuberculosis and HIV-co-infected patients: Th1 explosion or cytokine storm? AIDS 21: 882-884, 2007.

27. Shankar MS, Aravindan AN, Sohal PM, et al. The prevalence of tuberculin sensitivity and anergy in chronic renal failure in an endemic area: Tuberculin test and the risk of post-transplant tuberculosis. Nephrol Dial Transplant 20: 2720-2724, 2005.

28. Abdelrahman M, Sinha AK, Karkar A. Tuberculosis in end-stage renal disease patients on hemodialysis. Hemodial Int 10: 360-364, 2006.

29. Passalent L, Khan K, Richardson R, Wang J, Dedier H, Gardam M. Detecting latent tuberculosis infection in hemodialysis patients: A head-to-head comparison of the T-SPOT.TB test, and an expert physician panel. Clin J Am Soc Nephrol 2: 68-73, 2007.

30. Hoffmann M, Tsinalis D, Vernazza P, Fierz W, Binet I. Assessment of an interferon-gamma release assay for the diagnosis of latent tuberculosis infection in haemodialysis patients. Swiss Med Wkly 140: 286-292, 2010.

31. Targeted tuberculin testing and treatment of latent tuberculosis infection. American Thoracic Society. MMWR Recomm Rep 49: 151, 2000.

32. Cellestis. QuantiFERON s-TB Gold package insert. [Internet]. [cited 2013 Dec 20]. Available from: http://www.cellestis.com/IR $\mathrm{M} /$ content/PI/QFT/2PK/AU.pdf

33. RayBiotech. RayBio Human IP-10 ELISA Kit User Manual. [Internet]. [cited 2014 May 15]. Available from: http://www.raybiotec h.com/files/manual/ELISA/ELH-IP10-001.pdf

34. Azzurri A, Sow OY, Amedei A, et al. IFN-gamma-inducible protein 10 and pentraxin 3 plasma levels are tools for monitoring inflammation and disease activity in Mycobacterium tuberculosis infection. Microbes Infect 7: 1-8, 2005.

35. Ruhwald M, Bjerregaard-Andersen M, Rabna P, Kofoed K, Eugen-Olsen J, Ravn P. CXCL10/IP-10 release is induced by incubation of whole blood from tuberculosis patients with ESAT-6, CFP10 and TB7.7. Microbes Infect 9: 806-812, 2007.

36. Turkish Ministry of Health Department of Tuberculosis Control. The Book of Tuberculosis Control in Turkey. Ankara, 2003: 10 (in Turkish). 
37. Segall L, Covic A. Diagnosis of tuberculosis in dialysis patients: current strategy. Clin J Am Soc Nephrol 5: 1114-1122, 2010.

38. Wang L, Turner MO, Elwood RK, Schulzer M, FitzGerald JM. A meta-analysis of the effect of Bacille Calmette Guerin vaccination on tuberculin skin test measurements. Thorax 57: 804-809, 2002.

39. Nahid P, Pai M, Hopewell PC. Advances in the diagnosis and treatment of tuberculosis. Proc Am Thorac Soc 3: 103-110, 2006.

40. Richeldi L, Losi M, D'Amico R, et al. Performance of tests for latent tuberculosis in different groups of immunocompromised patients. Chest 136: 198-204, 2009.

41. Winthrop KL, Nyendak $\mathrm{M}$, Calvet $\mathrm{H}$, et al. Inter-feron-gamma release assays for diagnosing mycobacterium tuberculosis infection in renal dialysis patients. Clin J Am Soc Nephrol 3: 1357-1363, 2008.

42. Seyhan EC, Sökücü S, Altin S, et al. Comparison of the QuantiFERON-TB Gold In-Tube test with the tuberculin skin test for de- tecting latent tuberculosis infection in hemodialysis patients. Transpl Infect Dis 12: 98-105, 2010.

43. Pai M, Kalantri S, Dheda K. New tools and emerging technologies for the diagnosis of tuberculosis: part I. Latent tuberculosis. Expert Rev Mol Diagn 6: 413-422, 2006.

44. Frahm M, Goswami ND, Owzar K, et al. Discriminating between latent and active tuberculosis with multiple biomarker responses. Tuberculosis 91: 250-256, 2011.

45. Chakera A, Bennett SC, Cornall RJ. A whole blood monokinebased reporter assay provides a sensitive and robust measurement of the antigen-specific $\mathrm{T}$ cell response. J Translat Med 9: 143, 2011.

46. Petrucci R, Abu Amer N, Gurgel RQ, et al. Interferon gamma, interferon-gamma-induced-protein 10, and tuberculin responses of children at high risk of tuberculosis infection. Pediatr Infect Dis J 27: 1073-1077, 2008.

(C) 2015 The Japanese Society of Internal Medicine http://www.naika.or.jp/imonline/index.html 